OPEN ACCESS

Edited by:

Anna Rosell,

Vall d'Hebron Research Institute (NHIR), Spain

Reviewed by: Alejandro Bustamante, Vall d'Hebron University Hospital, Spain

Gerrit M. Grosse

Hannover Medical School, Germany

${ }^{*}$ Correspondence:

Catherine Vergely cvergely@u-bourgogne.fr

† Last co-authors

Specialty section: This article was submitted to Stroke,

a section of the journal Frontiers in Neurology

Received: 15 February 2019 Accepted: 24 May 2019

Published: 14 June 2019

Citation: Brenière C, Méloux A, Pédard M Marie $C$, Thouant $P$, Vergely $C$ and Béjot $Y$ (2019) Growth Differentiation Factor-15 (GDF-15) Is Associated With Mortality in Ischemic Stroke Patients Treated With Acute Revascularization Therapy. Front. Neurol. 10:611. doi: 10.3389/fneur.2019.00611

\section{Growth Differentiation Factor-15 (GDF-15) Is Associated With Mortality in Ischemic Stroke Patients Treated With Acute Revascularization Therapy}

\author{
Céline Brenière ${ }^{1,2}$, Alexandre Méloux ${ }^{1}$, Martin Pédard ${ }^{3}$, Christine Marie $^{3}$, \\ Pierre Thouant ${ }^{2,4}$, Catherine Vergely ${ }^{1 * \dagger}$ and Yannick Béjot ${ }^{1,2+}$ \\ ${ }^{1}$ Equipe d'Accueil (EA 7460): Physiopathologie et Epidémiologie Cérébro-Cardiovasculaires (PEC2), University Bourgogne \\ Franche-Comté, UFR Sciences de Santé, Dijon, France, ${ }^{2}$ Department of Neurology, University Hospital of Dijon, Dijon, \\ France, ${ }^{3}$ INSERM UMR1093-CAPS, University Bourgogne Franche-Comté, UFR Sciences de Santé, Dijon, France, \\ ${ }^{4}$ Department of Neuroradiology, University Hospital of Dijon, Dijon, France
}

Background and Aims: Growth differentiation factor-15 (GDF-15) has been identified as a robust marker of developing cardiovascular disease, however, little is currently known about its prognostic value in stroke patients. In a context of growing interest to discover new biomarkers in stroke, we aimed to assess the association between circulating GDF-15 levels and three-month mortality in ischemic stroke patients treated with acute revascularization therapy.

Methods: 173 patients hospitalized for acute ischemic stroke and treated with either intravenous thrombolysis ( $n=99,57.2 \%)$, mechanical thrombectomy ( $n=41,23.4 \%)$ or combined therapy ( $n=33,19.1 \%$ ) were prospectively included. Baseline clinical and biological characteristics were recorded. Plasma GDF-15 levels were measured at admission (D0), and at $24 \mathrm{~h}, 3$ and 7 days. Clinical severity was assessed with the National Institutes of Health Stroke Scale (NIHSS) score, and vital status was obtained 3 months after the stroke.

Results: At 3 months post-stroke, 32 patients (18.5\%) had died. The deceased patients had higher D0 plasma GDF-15 levels (median [IQR]: 2,777 [1,769-5,446] vs. 1,460 [965-2,079] pg/mL, $P<0.001)$. In multivariable logistic regression analysis, D0 GDF-15 levels in the third tertile of the distribution were independently associated with mortality at 3 months $(\mathrm{OR}=3.71 ; 95 \% \mathrm{Cl}: 1.09-12.6, P=0.036)$, even after adjustment for confounding variables including clinical severity.

Conclusions: Our data show for the first time that GDF-15 plasma concentration at admission is independently associated with 3-month mortality in ischemic stroke patients treated with acute revascularization therapy. The pathophysiological mechanisms that could explain this association warrant further study.

Keywords: stroke, GDF15, mortality, thrombectomy, thrombolysis 


\section{INTRODUCTION}

Extensive research has contributed to the identification and routine use of several blood biomarkers in the field of cardiovascular and metabolic diseases, but the recognition of such biomarkers for ischemic stroke patients remains challenging. One potential biomarker is growth differentiation factor 15 (GDF-15), a cytokine belonging to the TGF- $\beta$ superfamily (1). GDF-15 is weakly expressed in several tissues under physiological conditions except in the placenta $(2,3)$. Blood GDF-15 concentrations tend to increase with age and they do not vary with sex in apparently healthy elderly individuals, free of major medical comorbidities $(4,5)$. Moreover, GDF15 plasma concentrations do increase in pathological situations and are associated with hypoxia, inflammation, oxidative stress, and oncogene activation. Elevated GDF-15 levels have also been closely associated with all-cause mortality (6-8). As a biomarker, GDF-15 has widely been studied in cardiovascular diseases (9) where it is considered as an independent marker of poor prognosis in patients with coronary artery disease (10-12), acute myocardial infarction (13-16), atrial fibrillation (17), and heart failure with preserved or reduced ejection fraction $(18,19)$. The cellular effects of GDF-15 may also vary according to context. For instance, GDF-15 expression increases in macrophages and may have proinflammatory effects in atherosclerosis $(20,21)$, whereas anti-inflammatory effects have been observed in acute diseases such as myocardial infarction (22). Moreover, GDF-15 is massively upregulated by nitrosative stress in cardiomyocytes subjected to simulated ischemia-reperfusion (23), and has been experimentally identified as a cardioprotective cytokine $(22,24)$. In contrast, there is little available data concerning GDF-15 involvement in ischemic stroke. In the brains of rats and mice, where GDF- 15 is physiologically expressed in the choroid plexus epithelium, cortical lesioning or cerebral ischemia has been shown to induce a significant increase of GDF-15 expression in the injured cortex $(25,26)$. Recent data from our research unit also revealed that ischemic stroke in rats leads to a rapid and persistent increase of GDF-15 in blood circulation (27). In a more clinical context, GDF-15 could be used as a biomarker to predict stroke risk in hypertensive patients (28) as well as mortality and stroke risk in atrial fibrillation (29). Circulating GDF-15 is also associated with subclinical brain injury (30). It has been proposed that GDF-15 could be associated with poor functional outcomes after ischemic stroke (31-33). However, whether GDF-15 could be a biomarker of mortality after acute ischemic stroke treated with acute revascularization therapy remains to be elucidated. The main objective of our study was therefore to assess the association between circulating levels of GDF-15 and threemonth all-cause mortality in ischemic stroke patients treated with thrombolysis and/or mechanical thrombectomy.

\section{METHODS}

\section{Study Population}

The PARADISE study (Prognosis After Revascularization therapy in the Dijon Ischemic Stroke Evaluation study; Clinical trial NCT02856074) is an ongoing hospital-based cohort of consecutive patients with acute ischemic stroke treated with either intravenous thrombolysis (IVT) and/or mechanical thrombectomy, and who were admitted to the intensive care stroke unit of the Dijon University Hospital (Burgundy, France). For the present study, analyses were conducted on patients included between January 2017 and September 2017 for whom blood samples were collected. For each patient, ischemic stroke was diagnosed by a senior stroke-trained neurologist based on symptoms and a clinical evaluation, and confirmed by brain imaging (either cerebral computed tomography or magnetic resonance imaging). Acute revascularization therapy was performed in routine practice and based on the physician's application of the current recommendations. Ischemic stroke was classified using TOAST (Trial of ORG 10172 in Acute Stroke Treatment) criteria (34), according to results of the diagnostic work-up performed during hospitalization.

\section{Data Collected and Outcome Measured}

At inclusion, demographics and vascular risk factors were recorded: hypertension (high blood pressure reported in a patient's medical history or patients taking antihypertensive treatment), diabetes mellitus (glucose level $\geq 7.8 \mathrm{mmol} / \mathrm{L}$ reported in the medical record or patients under insulin or oral hypoglycaemic agents), hypercholesterolemia (total cholesterol level $\geq 5.7 \mathrm{mmol} / \mathrm{L}$ reported in the medical history or patients treated with lipid-lowering therapy), and current smoking. History of stroke, transient ischemic attack (TIA), myocardial infarction, peripheral artery disease, heart failure, and atrial fibrillation were also recorded. Stroke severity was evaluated using the National Institutes of Health Stroke Scale (NIHSS) score at admission (Day 0, D0), at $24 \mathrm{~h}$ (Day 1, D1), and at discharge. C-reactive protein (CRP) and creatinine levels were obtained from routine blood samples at admission.

The primary outcome was all-cause mortality at 3 months. Information about vital status was systematically obtained from patients and/or their relatives by telephone.

\section{GDF-15 Measurement}

Prior to the beginning of the study, we evaluated GDF-15 in blood samples stored at $4^{\circ} \mathrm{C}$ for a maximum duration of $24 \mathrm{~h}$ to establish the good stability of the biomarker in standard hospital storage conditions. We also included a standard pool of plasma in each set of measurement so as to exclude possible inter-experiment variations. Furthermore, we did not observe any effects on GDF-15 concentrations of long-term storage of samples at $-80^{\circ} \mathrm{C}$ (9 months). For this study, $2 \mathrm{~mL}$ of blood were collected at admission as soon as possible after reperfusion therapies (Day 0, D0), 24h $( \pm 2 \mathrm{~h})$ after the first sample (Day 1 , D1), at 3 days (Day 3, D3), and at 7 days (Day 7, D7). All blood samples were then quickly centrifuged at 3,500 rpm at $4{ }^{\circ} \mathrm{C}$ for $5 \mathrm{~min}$. Plasma samples were stored in aliquots at $-80^{\circ} \mathrm{C}$ for a maximum of 3 months until assayed. GDF-15 levels were measured with an Enzyme-linked Immuno-Sorbent Assay (ELISA) Development kit from R\&D Systems (Lille, France) according to manufacturer protocol. The detection limit was $23.4 \mathrm{pg} / \mathrm{mL}$. The operators performing the measurements were blinded to treatment and patient outcomes. 


\section{Statistical Analyses}

Baseline characteristics were given as frequencies for categorical variables, and as means, standard deviation, medians and interquartile ranges for continuous variables. Proportions and mean values were compared (for patients alive vs. dead at 3 months) using the Chi-Square Test, the Fisher Exact test, and the Wilcoxon-Mann-Whitney test when appropriate. We used the Wilcoxon signed-rank test to compare the circulating levels of GDF-15 at the various points in time and the Spearman rank correlation analysis to assess the correlations between GDF-15 levels and clinical severity (NIHSS score), age, and all vascular risk factors and previous vascular diseases. A Bonferroni correction was used to account for multiple comparisons. Logistic regression analysis was used to evaluate the association

TABLE 1 | Characteristics of patients according to vital status at 3 months.

\begin{tabular}{|c|c|c|c|c|}
\hline & All patients $(n=173)$ & Alive $(n=141)$ & Dead $(n=32)$ & $P$ \\
\hline \multicolumn{5}{|l|}{ Age (years) } \\
\hline Median (IQR) & $76.9(66.3-85.1)$ & $75.9(65.7-82.9)$ & $85.4(73.1-88.5)$ & \\
\hline Second tertile $(n=58)$ & & $51(36.2 \%)$ & $7(21.9 \%)$ & 0.122 \\
\hline Third tertile $(n=57)$ & & 38 (26.9\%) & 19 (59.4\%) & $<0.001$ \\
\hline $\mathrm{NIHSS}$ at D1 $(n=171)$ & $7.5 \pm 6.8$ & $5.6 \pm 5.2$ & $16.8 \pm 7.1$ & $<0.001$ \\
\hline Etiology (TOAST classification) & & & & 0.039 \\
\hline Large artery & $26(15 \%)$ & $17(12 \%)$ & $9(28.1 \%)$ & \\
\hline Cardioembolic & $66(38.2 \%)$ & $50(35.5 \%)$ & $16(50 \%)$ & \\
\hline Small vessel disease & $3(1.7 \%)$ & $3(2.1 \%)$ & 0 & \\
\hline Other causes & $6(3.5 \%)$ & $6(4.3 \%)$ & 0 & \\
\hline Hypertension & 108 (62.4\%) & $84(59.6 \%)$ & 24 (75\%) & 0.020 \\
\hline Diabetes mellitus & $27(15.6 \%)$ & $20(14.2 \%)$ & 7 (21.9\%) & 0.279 \\
\hline Current smoking & $21(12.1 \%)$ & $20(14.2 \%)$ & $1(3.1 \%)$ & 0.084 \\
\hline Hypercholesterolemia & $58(33.5 \%)$ & 48 (34\%) & $10(31.3 \%)$ & 0.763 \\
\hline \multicolumn{5}{|l|}{ Previous vascular diseases } \\
\hline Previous stroke & $20(11.6 \%)$ & $13(9.2 \%)$ & $7(21.88 \%)$ & 0.037 \\
\hline Previous TIA & $6(3.5 \%)$ & $6(4.3 \%)$ & 0 & 0.250 \\
\hline Myocardial infarction & $13(7.5 \%)$ & $9(6.4 \%)$ & $4(12.5 \%)$ & 0.236 \\
\hline Peripheral artery disease & $7(4.1 \%)$ & $3(2.1 \%)$ & $4(12.5 \%)$ & 0.007 \\
\hline Heart failure & 17 (9.8\%) & $10(7.1 \%)$ & 7 (21.9\%) & 0.011 \\
\hline Atrial fibrillation & $29(16.8 \%)$ & $21(14.9 \%)$ & $8(32 \%)$ & 0.167 \\
\hline IV Thrombolysis only & 99 (57.2\%) & $85(60.3 \%)$ & $14(43.8 \%)$ & \\
\hline Thrombectomy only & $41(23.4 \%)$ & 31 (22\%) & $10(31.3 \%)$ & \\
\hline Combined therapy & $33(19.1 \%)$ & $25(17.7 \%)$ & $8(25 \%)$ & \\
\hline \multicolumn{5}{|l|}{ Biological data } \\
\hline \multicolumn{5}{|l|}{ GDF-15 D0 (pg/mL) } \\
\hline mean, SD & $2,101 \pm 1,644$ & $1,756 \pm 1,203$ & $3,622 \pm 2,353$ & $<0.001$ \\
\hline Median (IQR) & $1,629(1,030-2,457)$ & $1,460(965-2,079)$ & $2,777(1,769-5,446)$ & \\
\hline \multicolumn{5}{|l|}{ CRP (mg/L) } \\
\hline Median (IQR) & $13.5 \pm 23.9$ & $10.1 \pm 17.9$ & $28.2 \pm 38$ & 0.001 \\
\hline \multicolumn{5}{|l|}{ Creatinine $(\mu \mathrm{mol} / \mathrm{L})$} \\
\hline Median (IQR) & $72(61-89$ & $74(62-86)$ & $70(58-93)$ & 0.812 \\
\hline
\end{tabular}

$M C A$, middle cerebral artery; $A C h O A$, anterior choroidal artery; $A C A$, anterior cerebral artery; $P C A$, posterior cerebral artery. 
between GDF-15 and mortality at 3 months. GDF-15 levels were analyzed according to the tertiles of their distribution (third tertile vs. first and second tertiles) because they did not meet the normality assumption. In multivariable models, we introduced age, sex, and all baseline characteristics for which a $P$-value $<0.20$. Additionally, receiver operator characteristic (ROC) analysis was performed, and the c-statistic representing the area under the ROC curve for the models was evaluated. We compared discrimination of models with and without GDF-15 variables by calculating the integrated discrimination improvement (IDI). $P$-Values $<0.05$ were considered statistically significant. Statistical analyses were performed with STATA@13 software (StataCorp LP, College Station, Texas, USA).

\section{Ethics}

The PARADISE study was approved by the Institutional Review Board (CPP EST I: 2015/32; IDRCB: 2015-A01664-45). Oral informed consent was collected from the patients or their relatives according to French Legislation.

\section{RESULTS}

\section{Baseline Characteristics of Study Population}

Over the study period, 175 patients with acute ischemic stroke treated with revascularization therapy were enrolled; two were subsequently excluded because 3-month vital status could not be confirmed. The final cohort consisted of 173 patients (mean age $74.6 \pm 13.6$ years, $49 \%$ women): 99 (57.2\%) received IVT alone, $41(23.4 \%)$ underwent mechanical thrombectomy alone, and 33 (19.1\%) received a combined therapy.

At 3 months, 32 patients (18.5\%) had died. The baseline characteristics of patients grouped by vital status at 3 months are shown in Table 1. Patients who died were older, and were more likely to have hypertension, history of stroke, peripheral artery disease, and heart failure. They had a greater NIHSS score at admission and at $24 \mathrm{~h}$, and higher levels of CRP at admission than surviving patients. In addition, data about premorbid Rankin score (mRS) was available for 162/173 (93.6\%) patients, including $138 / 141(97.8 \%)$ patients alive at 3 months, and 24/32 (75\%) patients dead at 3 months. Among overall patients, $25.9 \%$ had a prior-to-stroke mRS score $\geq 2(21 \%$ in patients alive at 3 months vs. $54.2 \%$ in dead patients, $p=0.001)$.

\section{GDF-15 Levels According to Clinical Characteristics and Time Trends}

GDF-15 levels at admission (D0) were correlated with age (Spearman's correlation coefficient $=0.49 ; P<0.001$ ), initial NIHSS score (Spearman's correlation coefficient $=0.32 ; P$ $=0.003$ ) (Figure 1), hypertension (Spearman's correlation coefficient $=0.38 ; P<0.001$ ), and diabetes (Spearman's correlation coefficient $=0.33 ; P<0.001$ ). In addition, GDF15 levels at D1 were also correlated with NIHSS score at $24 \mathrm{~h}$ (Spearman's correlation coefficient $=0.37 ; P<0.001 ; n=165$ ). GDF-15 circulating levels changed over time as follows: median [IQR] GDF-15 levels declined between D0 (1,629 [1,030-2,457] $\mathrm{pg} / \mathrm{mL})$ and D1 $(1,416[921-1,957] \mathrm{pg} / \mathrm{mL})(P<0.001 ; n=163)$; between D0 and D3 $(1,232[841-1,951] \mathrm{pg} / \mathrm{mL})(P<0.001 ; n=$
124), and between D0 and D7 $(1,285[836-1,892] \mathrm{pg} / \mathrm{mL})(P=$ 0.019; $n=45$ ) (Figure 2).

\section{Association Between GDF-15 Levels and Mortality at 3 Months}

In patients who had died at 3 months, median plasma GDF-15 levels were greater at D0 $(P<0.001)$, D1 $(P<0.001)$, D3 $(P<$ $0.001)$, and $\mathrm{D} 7(P=0.041)$ (Figure 3). Distribution of $\mathrm{mRS}$ scores at 3 months (Figure 4) shows that patients in the third (highest) tertile of D0 GDF-15 were more likely to be dead ( 38.8 vs. $8.6 \%$, $p$ $<0.001)$. In univariate logistic regression analysis, GDF-15 levels in the third tertile of the distribution at all times points were associated with a higher risk of death (Table 2). In multivariable logistic regression analysis, D0 GDF-15 levels in the third tertile of the distribution were associated with mortality at 3 months (OR $=3.71$; 95\% CI: 1.09-12.6, $p=0.036$ ) (Table 3). Addition of the premorbid mRS into the models did not alter the results. Hence, D0 GDF-15 levels in the third tertile of the distribution were associated with mortality at 3 months $(\mathrm{OR}=4.46$; $95 \% \mathrm{CI}$ : $1.15-17.4, p=0.031)$. No significant association between either D1 GDF-15 levels (OR $=0.33$; 95\% CI: 0.07-1.62, $p=0.17)$ or D3 GDF-15 levels (OR $=0.18$; 95\% CI: $0.01-3.71, p=0.27)$ and mortality were noted.

The model without including GDF-15 showed a good discrimination (c-statistic $=0.877 ; 95 \% \mathrm{CI}: 0.814-0.940)$ that was only slightly improved when adding GDF-15 (c-statistic $=0.891$; 95\% CI: 0.835-0.947) (IDI 0.023, $p=0.23$ ).

\section{DISCUSSION}

This study demonstrated that, in patients hospitalized for acute ischemic stroke who received IV thrombolysis and/or mechanical thrombectomy, higher concentrations of GDF-15 at admission were associated with increased risk of death at 3 months.

We sequentially measured GDF-15 from admission to 7 days after the acute ischemic event and we observed that GDF-15 levels decreased over this period, mainly between the 24 first hours. Our results are consistent with two other studies in which GDF-15 levels declined between $6 \mathrm{~h}$ and 7 days after ischemic stroke in 57 patients (31) and between admission and $24 \mathrm{~h}$ in 264 patients (32). While we have no data concerning the pre-ischemic levels of GDF-15 in these stroke patients, we can speculate that GDF-15 concentrations were certainly elevated as a result of age and the associated cardiovascular risk factors such as hypertension and diabetes that were shown to be determinants of circulating GDF-15 levels in a large cohort study (5). As a matter of comparison, while mean GDF-15 levels in young healthy adults are around $450 \mathrm{pg} / \mathrm{ml}$ (unpublished data from preliminary experiments), the mean concentrations at admission and during hospitalization in the stroke patients presented here were approximately four times higher $(2,100 \pm$ 125 at D0 and 1,886 \pm 269 at D7, mean \pm SEM). Additionally, we cannot exclude the possibility that GDF-15 levels spiked during the acute cerebrovascular event as a result of ischemia and inflammation. Recent experimental data from our team revealed that ischemic stroke in rats prompted the increase in GDF-15 


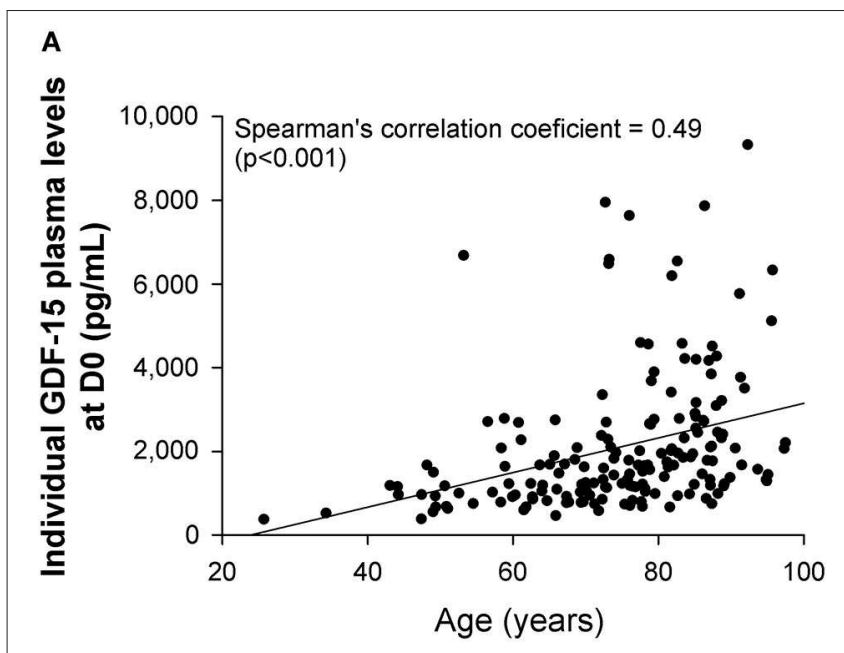

B

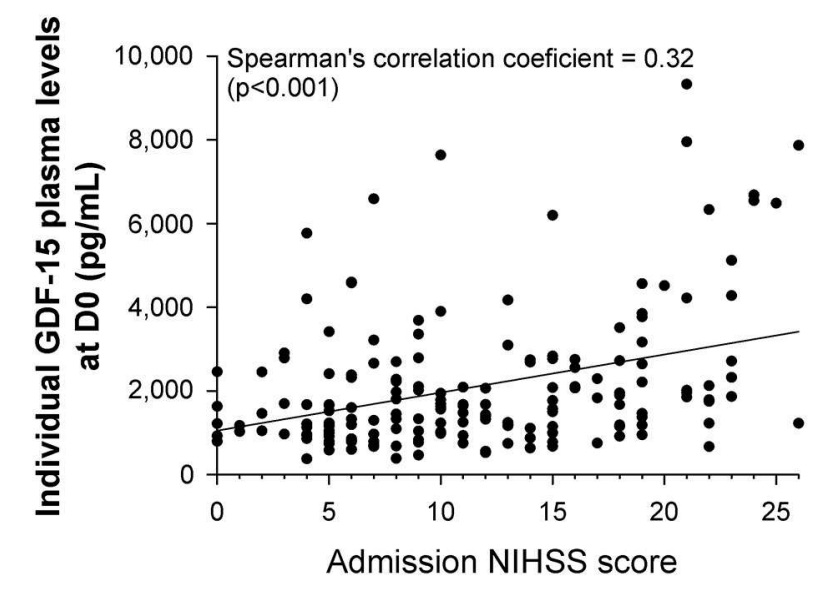

FIGURE 1 | Correlation between individual GDF-15 plasma levels at admission (D0) and either (A) age or (B) admission NIHSS score $(n=173)$.

in the circulation 2 and $24 \mathrm{~h}$ after cerebral embolization (27). In pathological conditions, GDF-15 may originate from a number of tissues and in particular from inflammatory cells. However, it has been shown that cryogenic cerebral lesions induced GDF15 expression in macrophages, neurons and microglial cells (25). Other data have also shown the induction of GDF-15 mRNA expression in the ipsilateral hippocampus and parietal cortex 3 and $24 \mathrm{~h}$ after cerebral ischemia induced by middle cerebral artery occlusion in mice (26). Our team has made similar unpublished observations in the ipsilateral cortex and cerebellum after cerebral embolization in rats. Therefore, we can hypothesize that the increased GDF-15 concentration in stroke patients may originate both from the pre-ischemic context of high risk factors in an elderly population and from the acute ischemic inflammatory event. Moreover, in line with a previous study (31), we found that GDF-15 levels were correlated with NIHSS score that is an appropriate rating scale for clinical severity. Our findings therefore suggest that elevated GDF-15 levels may be associated with higher clinical severity in concordance with more serious ischemic lesions.

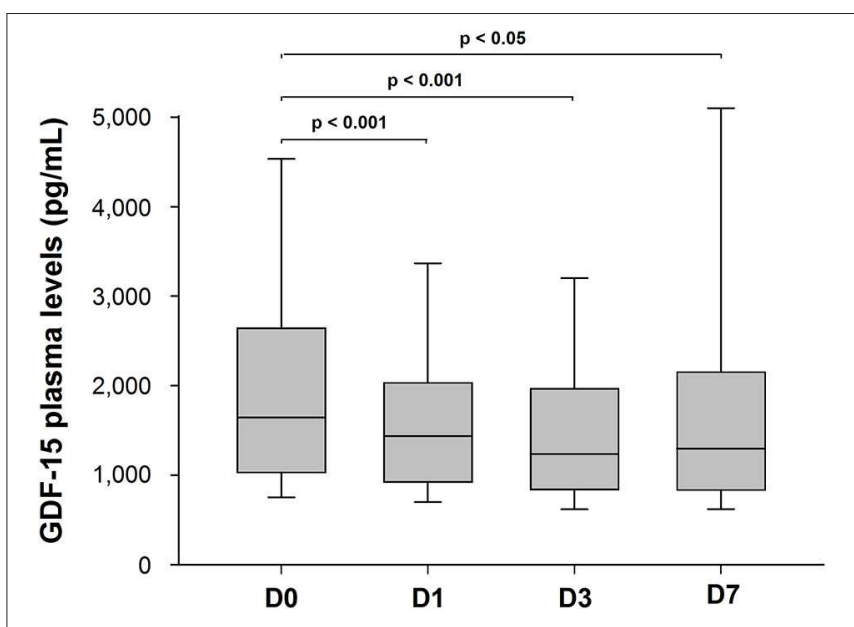

FIGURE 2 | Time trends in median GDF-15 plasma levels of stroke patients at D0 $(n=173)$, D1 $(n=163)$, D3 $(n=124)$, and D7 $(n=45)$ after admission.

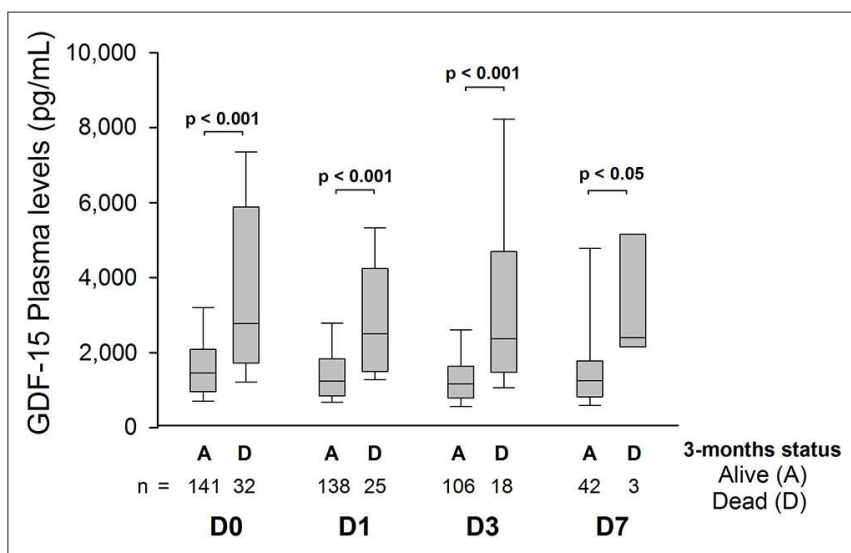

FIGURE 3 | Median GDF-15 plasma levels at D0 ( $n=173)$, D1 $(n=163)$, D3 $(n=124)$, and D7 $(n=45)$ according to vital status at 3 months.

Our study shows that GDF-15 levels at admission are associated with post-stroke mortality even after adjustment for other usual predictors, although the improvement of prediction compared with a clinical model was only limited. GDF-15 has previously been investigated as a potential biomarker of global mortality. For instance, in 1,004 elderly individuals, changes in GDF-15 concentrations between 70 and 75 years were predictors of all-cause mortality (7). In 940 71-year-old subjects followed for 10 years, an increase in GDF-15 levels was associated with a stronger risk of cardiovascular mortality, total mortality and coronary-heart-disease-related morbidity and mortality (35). GDF-15 has been recognized as a biomarker of mortality and cardiovascular events in patients with STelevation or non-ST-elevation acute coronary syndromes (29). In fourteen thousand five hundred and seventy-seven patients with stable coronary heart disease, the highest quartile of GDF15 concentrations was highly associated with all-cause morbidity and with stroke (12). We observed that GDF-15 levels at admission were associated with post-stroke mortality even after adjustment for multiple variables such as NIHSS score which is 


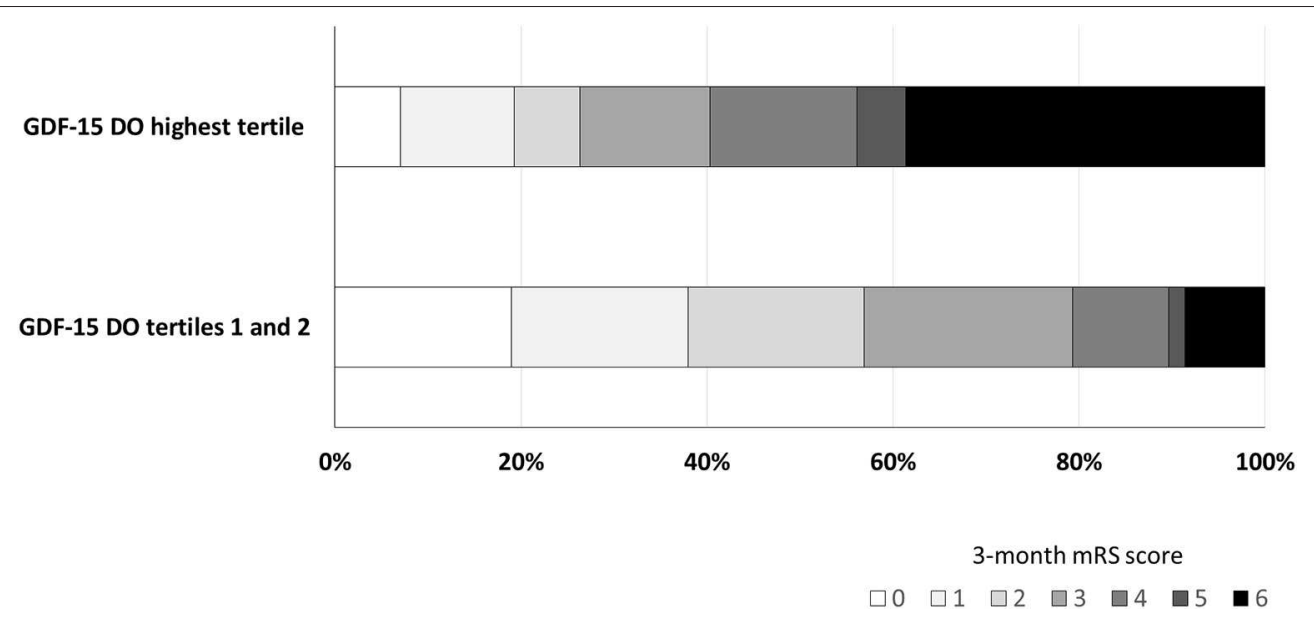

FIGURE 4 | Distribution of mRS scores at 3 months according to D0 GDF-15 tertiles.

TABLE 2 | Association between 3-month mortality and GDF-15 levels in the third tertile at D0 (GDF > 2,088 pg/mL), D1 (GDF > 1,784 pg/mL), and D3 (GDF $>1,575 \mathrm{pg} / \mathrm{mL}$ ) in univariate logistic regression analysis.

\begin{tabular}{lccc}
\hline & OR & $\mathbf{9 5 \% ~ C l ~}$ & $\boldsymbol{P}$-value \\
\hline GDF-15 D0 $(n=173)$ & 6.66 & $2.88-15.43$ & $<0.001$ \\
GDF-15 D1 $(n=163)$ & 3.81 & $1.58-9.20$ & 0.003 \\
GDF-15 D3 $(n=124)$ & 5.31 & $1.82-15.47$ & 0.002 \\
\hline
\end{tabular}

TABLE 3 | Multivariable logistic regression analysis of the association between 3-month mortality and GDF-15 levels in the third tertile of the distribution at D0 (GDF-15 > 2,088 pg/mL), D1 (GDF-15 > 1,784 pg/mL), and D3 (GDF-15 > 1,575 $\mathrm{pg} / \mathrm{mL})$.

\begin{tabular}{lccc}
\hline & OR $^{\S}$ & 95\% Cl & $P$-value \\
\hline GDF-15 D0 $(n=173)$ & 3.71 & $1.09-12.62$ & 0.036 \\
GDF-15 D1 $(n=163)$ & 0.62 & $0.16-2.34$ & 0.478 \\
GDF-15 D3 $(n=124)$ & 0.62 & $0.77-4.97$ & 0.650 \\
\hline
\end{tabular}

\$Adjusted for age, sex, NIHSS score at admission, hypertension, smoking, previous stroke, peripheral artery disease, heart failure, atrial fibrillation, CRP levels (third tertile), and acute revascularization therapy.

a very useful scale for prognosis evaluation after stroke (36) and even after adjustment for other usual predictors. This finding is consistent with the recently published study on 3,066 ischemic stroke patients in which high GDF-15 levels were associated with unfavorable clinical outcomes including death, and major disability (33). GDF-15 may therefore have a predictive power in addition to clinical and biologic variables for the mortality risk evaluation after ischemic stroke. Our findings suggest that admission GDF-15 may be an additional biomarker to predict the outcomes of ischemic stroke patients who specifically receive acute revascularization therapy.

One of the strengths of our study is that we assessed the stability of GDF-15 levels both in blood samples stored at $4^{\circ} \mathrm{C}$ for a maximum duration of $24 \mathrm{~h}$ and in frozen plasma aliquots stored for a long-term at $-80^{\circ} \mathrm{C}$. Besides, in previous studies aimed at discovering different prognosis biomarkers after stroke, most of them included small groups and did not adjust for clinical information such as NIHSS or age (37). Several limitations must be acknowledged. Since samples were collected at various times after reperfusion therapies, we cannot exclude a possible source of variability due to the administration of exogenous tPA. It was not possible to clearly establish whether GDF-15 levels increase with stroke volume since no systematic MRI assessment was done. In addition, cause of death and delay of death were not recorded, thus making it impossible to specifically investigate the role of GDF-15 levels on mortality. Although the deleterious impact of elevated GDF-15 on bleeding risk has been documented, symptomatic haemorrhagic transformation was observed in only a few number of patients ( 2 in surviving patients and 4 in dead patients), that cannot allow to draw any definite conclusions.

To conclude, our study is the first providing data on GDF-15 time course in the plasma of post-stroke patients undergoing revascularization, either by thrombolysis or mechanical thrombectomy. Our findings further suggest that the GDF-15 cytokine could be a prognostic biomarker of mortality in ischemic stroke patients treated with acute revascularization therapy. Further studies are needed to better understand the pathophysiological mechanisms that could explain this association.

\section{DISCLOSURE}

YB: honoraria for lectures or consulting fees from AstraZeneca, Daiichi-Sankyo, BMS, Pfizer, Medtronic, MSD France, Amgen, and Boehringer-Ingelheim. CB, MP, CM, AM, Luc Rochette, CV: None.

\section{DATA AVAILABILITY}

The raw data supporting the conclusions of this manuscript will be made available by the authors, without undue reservation, to any qualified researcher. 


\section{ETHICS STATEMENT}

The PARADISE study was approved by the Institutional Review Board (CPP EST I: 2015/32; IDRCB: 2015-A01664-45). Oral informed consent was collected from the patients or their relatives according to French Legislation.

\section{AUTHOR CONTRIBUTIONS}

CB: data collection, sample analysis, article writing. AM and MP: sample analysis. PT and CM: data collection and analysis. CV and YB: study design, data analysis, article writing.

\section{REFERENCES}

1. Ago T, Sadoshima J. GDF15, a cardioprotective TGF-beta superfamily protein. Circ Res. (2006) 98:294-7. doi: 10.1161/01.RES.0000207919.83894.9d

2. Hromas R, Hufford M, Sutton J, Xu D, Li Y, Lu L. PLAB, a novel placental bone morphogenetic protein. Biochim Biophys Acta. (1997) 1354:40-4.

3. Moore AG, Brown DA, Fairlie WD, Bauskin AR, Brown PK, Munier ML, et al. The transforming growth factor-ss superfamily cytokine macrophage inhibitory cytokine- 1 is present in high concentrations in the serum of pregnant women. J Clin Endocrinol Metab. (2000) 85:4781-8. doi: $10.1210 /$ jcem.85.12.7007

4. Kempf T, Horn-Wichmann R, Brabant G, Peter T, Allhoff T, Klein G, et al. Circulating concentrations of growth-differentiation factor 15 in apparently healthy elderly individuals and patients with chronic heart failure as assessed by a new immunoradiometric sandwich assay. Clin Chem. (2007) 53:284-91. doi: $10.1373 /$ clinchem. 2006.076828

5. Ho JE, Mahajan A, Chen MH, Larson MG, McCabe EL, Ghorbani $\mathrm{A}$, et al. Clinical and genetic correlates of growth differentiation factor 15 in the community. Clin Chem. (2012) 58:1582-91. doi: 10.1373 /clinchem.2012.190322

6. Wiklund FE, Bennet AM, Magnusson PK, Eriksson UK, Lindmark F, Wu L, et al. Macrophage inhibitory cytokine-1 (MIC-1/GDF15): a new marker of all-cause mortality. Aging Cell. (2010) 9:1057-64. doi: 10.1111/j.1474-9726.2010.00629.x

7. Eggers KM, Kempf T, Wallentin L, Wollert KC, Lind L. Change in growth differentiation factor 15 concentrations over time independently predicts mortality in community-dwelling elderly individuals. Clin Chem. (2013) 59:1091-8. doi: 10.1373/clinchem.2012.201210

8. Kahli A, Guenancia C, Zeller M, Grosjean S, Stamboul K, Rochette L, et al. Growth differentiation factor-15 (GDF-15) levels are associated with cardiac and renal injury in patients undergoing coronary artery bypass grafting with cardiopulmonary bypass. PLoS ONE. (2014) 9:e105759. doi: 10.1371/journal.pone.0105759

9. Wollert KC, Kempf T, Wallentin L. Growth differentiation factor 15 as a biomarker in cardiovascular disease. Clin Chem. (2017) 63:140-51. doi: 10.1373/clinchem.2016.255174

10. Eggers KM, Kempf T, Allhoff T, Lindahl B, Wallentin L, Wollert KC. Growthdifferentiation factor- 15 for early risk stratification in patients with acute chest pain. Eur Heart J. (2008) 29:2327-35. doi: 10.1093/eurheartj/ehn339

11. Schaub N, Reichlin T, Twerenbold R, Reiter M, Steuer S, Bassetti S, et al. Growth differentiation factor-15 in the early diagnosis and risk stratification of patients with acute chest pain. Clin Chem. (2012) 58:441-9. doi: 10.1373/clinchem.2011.173310

12. Hagstrom E, Held C, Stewart RA, Aylward PE, Budaj A, Cannon CP, et al. Growth differentiation factor 15 predicts all-cause morbidity and mortality in stable coronary heart disease. Clin Chem. (2017) 63:325-33. doi: 10.1373/clinchem.2016.260570

13. Kempf T, Bjorklund E, Olofsson S, Lindahl B, Allhoff $\mathrm{T}$, Peter $\mathrm{T}$, et al. Growth-differentiation factor-15 improves risk stratification in STsegment elevation myocardial infarction. Eur Heart J. (2007) 28:2858-65. doi: 10.1093/eurheartj/ehm465

\section{FUNDING}

This study was supported by funding from the French Ministry of Research, from the Société Française Neurovasulaire, the Regional Council of Burgundy, the Dijon University Hospital and Dijon Faculty of Heath Sciences.

\section{ACKNOWLEDGMENTS}

The authors thank Suzanne Rankin for English revision of the manuscript, Ivan Porcherot for technical assistance, Jérôme Durier for statistical analysis assistance.

14. Wollert KC, Kempf T, Peter T, Olofsson S, James S, Johnston N, et al Prognostic value of growth-differentiation factor-15 in patients with nonST-elevation acute coronary syndrome. Circulation. (2007) 115:962-71. doi: 10.1161/CIRCULATIONAHA.106.650846

15. Khan SQ, Ng K, Dhillon O, Kelly D, Quinn P, Squire IB, et al. Growth differentiation factor-15 as a prognostic marker in patients with acute myocardial infarction. Eur Heart J. (2009) 30:1057-65. doi: 10.1093/eurheartj/ehn600

16. Widera C, Pencina MJ, Meisner A, Kempf T, Bethmann K, Marquardt I, et al. Adjustment of the GRACE score by growth differentiation factor 15 enables a more accurate appreciation of risk in non-ST-elevation acute coronary syndrome. Eur Heart J. (2012) 33:1095-104. doi: 10.1093/eurheartj/ehr444

17. Wallentin L, Hijazi Z, Andersson U, Alexander JH, De Caterina R, Hanna $\mathrm{M}$, et al. Growth differentiation factor 15 , a marker of oxidative stress and inflammation, for risk assessment in patients with atrial fibrillation: insights from the Apixaban for Reduction in Stroke and Other Thromboembolic Events in Atrial Fibrillation (ARISTOTLE) trial. Circulation. (2014) 130:184758. doi: 10.1161/CIRCULATIONAHA.114.011204

18. Kempf T, von Haehling S, Peter T, Allhoff T, Cicoira M, Doehner W, et al. Prognostic utility of growth differentiation factor-15 in patients with chronic heart failure. J Am Coll Cardiol. (2007) 50:1054-60. doi: 10.1016/j.jacc.2007.04.091

19. Chan MM, Santhanakrishnan R, Chong JP, Chen Z, Tai BC, Liew OW, et al. Growth differentiation factor 15 in heart failure with preserved vs. reduced ejection fraction. Eur J Heart Fail. (2016) 18:81-8. doi: 10.1002/ejhf.431

20. Schlittenhardt D, Schober A, Strelau J, Bonaterra GA, Schmiedt W, Unsicker $\mathrm{K}$, et al. Involvement of growth differentiation factor- $15 /$ macrophage inhibitory cytokine-1 (GDF-15/MIC-1) in oxLDL-induced apoptosis of human macrophages in vitro and in arteriosclerotic lesions. Cell Tissue Res. (2004) 318:325-33. doi: 10.1007/s00441-004-0986-3

21. de Jager SC, Bermudez B, Bot I, Koenen RR, Bot M, Kavelaars A, et al. Growth differentiation factor 15 deficiency protects against atherosclerosis by attenuating CCR2-mediated macrophage chemotaxis. J Exp Med. (2011) 208:217-25. doi: 10.1084/jem.20100370

22. Kempf T, Zarbock A, Widera C, Butz S, Stadtmann A, Rossaint J, et al. GDF-15 is an inhibitor of leukocyte integrin activation required for survival after myocardial infarction in mice. Nat Med. (2011) 17:581-8. doi: $10.1038 / \mathrm{nm} .2354$

23. Kempf T, Eden M, Strelau J, Naguib M, Willenbockel C, Tongers J, et al. The transforming growth factor-beta superfamily member growth-differentiation factor-15 protects the heart from ischemia/reperfusion injury. Circ Res. (2006) 98:351-60. doi: 10.1161/01.RES.0000202805.73038.48

24. Xu J, Kimball TR, Lorenz JN, Brown DA, Bauskin AR, Klevitsky R, et al. GDF15/MIC-1 functions as a protective and antihypertrophic factor released from the myocardium in association with SMAD protein activation. Circ Res. (2006) 98:342-50. doi: 10.1161/01.RES.0000202804.8 4885.d0

25. Schober A, Bottner M, Strelau J, Kinscherf R, Bonaterra GA, Barth M, et al. Expression of growth differentiation factor-15/ macrophage inhibitory cytokine-1 (GDF-15/MIC-1) in the perinatal, adult, and injured rat brain. $J$ Comp Neurol. (2001) 439:32-45. doi: 10.1002/cne.1333 
26. Schindowski K, von Bohlen und Halbach O, Strelau J, Ridder DA, Herrmann O, Schober A, et al. Regulation of GDF-15, a distant TGF-beta superfamily member, in a mouse model of cerebral ischemia. Cell Tissue Res. (2011) 343:399-409. doi: 10.1007/s00441-010-1090-5

27. Meloux A, Rigal E, Rochette L, Cottin Y, Bejot Y, Vergely C. Ischemic stroke increases heart vulnerability to ischemia-reperfusion and alters myocardial cardioprotective pathways. Stroke. (2018) 49:2752-60. doi: 10.1161/STROKEAHA.118.022207

28. Wang X, Zhu L, Wu Y, Sun K, Su M, Yu L, et al. Plasma growth differentiation factor 15 predicts first-ever stroke in hypertensive patients. Medicine. (2016) 95:e4342. doi: 10.1097/MD.0000000000004342

29. Hagstrom E, James SK, Bertilsson M, Becker RC, Himmelmann A, Husted S, et al. Growth differentiation factor-15 level predicts major bleeding and cardiovascular events in patients with acute coronary syndromes: results from the PLATO study. Eur Heart J. (2016) 37:1325-33. doi: 10.1093/eurheartj/ehv491

30. Andersson C, Preis SR, Beiser A, DeCarli C, Wollert KC, Wang TJ, et al. Associations of circulating growth differentiation factor-15 and ST2 concentrations with subclinical vascular brain injury and incident stroke. Stroke. (2015) 46:2568-75. doi: 10.1161/STROKEAHA.115.009026

31. Worthmann H, Kempf T, Widera C, Tryc AB, Goldbecker A, Ma YT, et al. Growth differentiation factor 15 plasma levels and outcome after ischemic stroke. Cerebrovasc Dis. (2011) 32:72-8. doi: 10.1159/000328233

32. Groschel K, Schnaudigel S, Edelmann F, Niehaus CF, Weber-Kruger M, Haase $\mathrm{B}$, et al. Growth-differentiation factor-15 and functional outcome after acute ischemic stroke. J Neurol. (2012) 259:1574-9. doi: 10.1007/s00415-011-6379-0

33. Yin J, Zhu Z, Guo D, Wang A, Zeng $N$, Zheng $X$, et al. Increased growth differentiation factor 15 is associated with unfavorable clinical outcomes of acute ischemic stroke. Clin Chem. (2019) 65:569-78. doi: 10.1373/clinchem.2018.297879

34. Adams HP Jr., Bendixen BH, Kappelle LJ, Biller J, Love BB, Gordon DL. (1993). Classification of subtype of acute ischemic stroke. Definitions for use in a multicenter clinical trial. TOAST. Trial of Org 10172 in Acute Stroke Treatment. Stroke. 24:35-41.

35. Wallentin L, Zethelius B, Berglund L, Eggers KM, Lind L, Lindahl $\mathrm{B}$, et al. GDF-15 for prognostication of cardiovascular and cancer morbidity and mortality in men. PLoS ONE. (2013) 8:e78797. doi: 10.1371/journal.pone.0078797

36. Kasner SE. Clinical interpretation and use of stroke scales. Lancet Neurol. (2006) 5:603-12. doi: 10.1016/S1474-4422(06)70495-1

37. Whiteley W, Chong WL, Sengupta A, Sandercock P. Blood markers for the prognosis of ischemic stroke: a systematic review. Stroke. (2009) 40:e380-9. doi: 10.1161/STROKEAHA.108. 528752

Conflict of Interest Statement: The authors declare that the research was conducted in the absence of any commercial or financial relationships that could be construed as a potential conflict of interest.

Copyright (C) 2019 Brenière, Méloux, Pédard, Marie, Thouant, Vergely and Béjot. This is an open-access article distributed under the terms of the Creative Commons Attribution License (CC BY). The use, distribution or reproduction in other forums is permitted, provided the original author(s) and the copyright owner(s) are credited and that the original publication in this journal is cited, in accordance with accepted academic practice. No use, distribution or reproduction is permitted which does not comply with these terms. 